\title{
LA GENERACIÓN DE CONOCIMIENTO COMO PROYECTO Y ACCIÓN COLECTIVA
}

\section{[EN] THE GENERATION OF KNOWLEDGE AS A PROJECT AND COLLECTIVE ACTION}

La revista Voces desde el Trabajo Social [RVTS] representa la concreción de un proyecto colectivo que, desde sus inicios en el 2012, ha buscado contribuir y ampliar aquellos espacios a los que tradicionalmente se ha relacionado la generación de conocimiento y accionar académico. De esta forma, la RVTS se encausa a partir de un conjunto de ideas-faro, las cuales nos posibiliten ejercer como conectores y facilitadores del diálogo entre el campo de lo social y la comunidad académica y profesional a nivel nacional, regional e internacional. Particularmente, esto hace referencia a promover el intercambio enriquecedor a través de experiencias y difusión de trabajos generados desde el Trabajo Social y las ciencias sociales y humanas en general. Esto pone sobre la mesa lo mucho que tenemos para aprender de otras disciplinas y profesiones a fines, pero, desde el Trabajo Social jes tanto lo que tenemos para enunciar, compartir y construir!

Con la publicación de este primer editorial en la RVTS, se pone en marcha una serie de cambios, entre los que incluye la inauguración de su nuevo formato digital y la incorporación de diversas secciones, tales como artículos de investigación y revisión, historia y subjetividades, reseñas críticas de textos, presentación de libros, notas del Trabajo Social y proyectos creativos. También, se 
conformó una nueva estructura editorial y logró la indexación de la revista en diversos espacios reconocidos a nivel internacional. Dicho recorrido se encuentra iluminado por los principios de pluralidad, interdisciplinariedad, buenas prácticas académicas y las más altas consideraciones éticas. Sin lugar a duda, los anteriores enunciados dejan entrever nuestra aspiración de tornar la RVTS como parte de un tejido y red del conocimiento, lo que en palabras de Derrida (2018) es la tarea para la [de]construcción. Pero también, incorporando la dinámica, gestión y de aquello que Martínez y Agüero (2014) y Najmanovich (2005) llaman indisciplina, o sea, rebeldía, interpelación y cuestionamiento.

Invito a todas las personas lectoras de la RVTS a que examinen y compartan este volumen, el cual resulta de diversos esfuerzos. Definitivamente, es gracias al apoyo de diversas personas que estuvieron dispuestas para asumir este reto de edificar conjuntamente. Especialmente, destaco el papel fundamental de la Junta Directiva del Colegio de Profesionales del Trabajo Social de Puerto Rico [CPTSPR], Junta Editorial, persona de soporte digital, la Dirección Ejecutiva del CPTSPR y a todas las autoras y autores que presentaron sus valiosos trabajos en nuestra revista. También, hago extensivos mis profundos agradecimientos a la Junta Asesora Internacional y el Consejo Asesor Interdisciplinario de la RVTS quienes, sin dudarlo, reafirmaron su compromiso intelectual y dijeron sí a formar parte en nuestro proyecto. De igual forma, acentúo el apoyo derivado por personal académico de la Escuela Graduada de Ciencias y Tecnologías de la Información de la Universidad de Puerto Rico [EGCTI-UPR].

Como parte de mis palabras finales, insto a que puedan aprovechar al máximo aquellas herramientas y enlaces integrados a la versión digital de la RVTS. Adicionalmente, hago extensiva la invitación 
para que formen parte de este proyecto colectivo, que tal y como mencioné al inicio, se constituye como algo posible en la medida en que se amplíe la integración, participación y sentido de pertenencia de esta, su revista. En nombre de la Junta Editorial, deseamos que esta publicación represente una contribución que, configurada desde Puerto Rico, se abre paso a otras latitudes.

\section{Referencias}

Derrida, J. (2018). Theory and practice. The seminars of Jacques Derrida. Chicago: University of Chicago Press.

Martínez, S., \& Agüero, J. (2014). Trabajo Social Emancipador. De la disciplina a la indisciplina. Paraná: Hendija.

Najmanovich, D. (2005). El juego de los vínculos. Subjetividad y redes: Figuras en mutación. Buenos Aires: Biblos.

\section{${ }^{1}$ Eduardo Zavala-Mendoza}

Editor General del Colegio de Profesionales del Trabajo Social de Puerto Rico.

Profesor Conferenciante, Escuela de Ciencias Sociales y Humanas de la Universidad de Este, Campus Carolina, Puerto Rico

\zavalae1@suagm.edu

(D) ORCID ID https://orcid.org/0000-0002-1909-7856 